\title{
Hidrólisis enzimática del pasto maralfalfa (Pennisetum sp) sometido a extrusión húmeda
}

Ligia Johana Jaimes Cruz; Cristian Adoni Menjivar Dominguez²; Elsy Valeska Montoya Almendarez; Esdras Omar Mendoza Orellana²; Héctor Jairo Correa Cardona ${ }^{3 *}$; Ángel Giraldo Mejía ${ }^{3}$;

Ángela Adriana Ruíz-Colorado ${ }^{4}$

${ }^{1}$ Estudiante de Doctorado en Ciencias Agrarias, Facultad de Ciencias Agrarias, Universidad Nacional de Colombia, sede Medellín; ${ }^{2}$ Universidad Nacional de Agricultura de Honduras; ${ }^{3}$ Departamento de Producción Animal, Facultad de Ciencias Agrarias, Universidad Nacional de Colombia, sede Medellín; ${ }^{4}$ Departamento de Procesos y Energía, Facultad de Minas, Universidad Nacional de Colombia, sede Medellín.

*hjcorreac@unal.edu.co

Fecha recepción: febrero 28 de 2020 Fecha aceptación: abril 16 de 2020

\section{Resumen}

Se evaluó el efecto de la extrusión del pasto maralfalfa (Pennisetum sp) sobre la composición química de la pared celular y la digestibilidad in vitro de la materia seca y de la fibra en detergente neutro. Para ello se recolectaron siete muestras $(10,0 \mathrm{~kg} /$ muestra) del mismo lote, con 51 días de rebrote y se picaron a $2 \mathrm{~cm}$. Tres de estas muestras, tomadas al azar, se procesaron frescas en un extrusor de tornillo cónico girando a $1050 \mathrm{rpm}$ y con salida de $3 \mathrm{~mm}$, mientras que las otras cuatro fueron extruidas en el mismo equipo con salida de $1 \mathrm{~mm}$. En las muestras de pasto crudo y en las de los bagazos procedentes de la extrusión se determinó el contenido de fibra en detergente neutro, fibra en detergente ácido, lignina en detergente ácido, digestibilidad in vitro de la materia seca y digestibilidad in vitro de la fibra en detergente neutro. Se aplicó la prueba de T de Student para analizar el efecto del tipo de tratamiento, tanto entre ellos como respecto al pasto fresco. Los resultados indican que, con relación al pasto fresco, la extrusión generó un bagazo con alto contenido de fibra en detergente neutro, incrementó la digestibilidad in vitro de la materia seca en $8,81 \%$ y la de la digestibilidad in vitro de la fibra en detergente neutro en $20,6 \%$, pero no diferencia con el tamaño de la salida del extrusor $(p<0,1)$. Se concluye que el proceso de extrusión como fue aplicado al pasto maralfalfa en este experimento mejora la digestibilidad de la materia seca y de la fibra en detergente neutro.

Palabras clave: Bagazo; Biomasa; Delignificación; Fibra; In vitro. 


\title{
Enzymatic hydrolysis of maralfalfa grass (Pennisetum sp) submmited to wet extrusion
}

\begin{abstract}
The effect of extrusion of maralfalfa grass (Pennisetum $s p$ ) on the chemical composition of the cell wall and the in vitro digestibility of dry matter and fiber in neutral detergent was evaluated. Seven samples $(10.0 \mathrm{~kg} / \mathrm{sample})$ were collected from the same batch, with 51 days of regrowth, and chopped $2 \mathrm{~cm}$. Three of them, taken at random, were processed fresh in a conical screw extruder turning at $1050 \mathrm{rpm}$ and with $3 \mathrm{~mm}$ output, while the other four were extruded in the same equipment with $1 \mathrm{~mm}$ output. In the samples of raw grass and bagasse from the extrusion, the fibre content in neutral detergent, fibre in acid detergent, lignin in acid detergent, in vitro digestibility of dry matter and in vitro digestibility fibre in neutral detergent were determined. The Student T test was applied to analyze the effect of the type of treatment, both among them and with respect to fresh grass. The results indicate that, with respect to fresh grass, the extrusion generated a bagasse with high fiber content in neutral detergent, increased the in vitro digestibility of dry matter by $8.81 \%$ and that of fiber in neutral detergent by $20.6 \%$, but did not differ due to the size of the extruder outlet $(p<0.1)$. It is concluded that the extrusion process as applied to maralfalfa grass in this experiment improves the digestibility of dry matter and fiber in neutral detergent.
\end{abstract}

Keywords: Bagasse; Biomass; Delignification; Fiber; In vitro.

\section{Hidrólise enzimática de capim- maralfalfa (Pennisetum sp) submetida a extrusão úmida}

\section{Resumo}

Foi avaliado o efeito da extrusão do capim maralfalfa (Pennisetum $s p$ ) na composição química da parede celular e a digestibilidade in vitro da matéria seca e da fibra em detergente neutro. Sete amostras (10,0 $\mathrm{kg} / \mathrm{amostra}$ ) foram coletadas do mesmo lote, com 51 dias de recrescimento, e picadas $2 \mathrm{~cm}$. Três deles, tomados ao acaso, foram processados frescos em uma extrusora de rosca cônica girando a $1050 \mathrm{rpm}$ e com saída de $3 \mathrm{~mm}$, enquanto os outros quatro foram no mesmo equipamento com saída de $1 \mathrm{~mm}$. Nas amostras do capim fresco e dos bagaços da extrusão, foram determinados o teor de fibra em detergente neutro, fibra em detergente ácido, lignina em detergente ácido, digestibilidade in vitro da matéria seca e fibra em detergente neutro. O teste $T$ de Student foi aplicado para analisar o efeito do tipo de tratamento, tanto entre eles como em relação ao capim fresco. Os resultados indicam que, em relação ao capim fresco, a extrusão gerou um bagaço com alto teor de fibra em detergente neutro, aumentou a digestibilidade in vitro da matéria seca em $8,81 \%$ e a da fibra em detergente neutro em $20,6 \%$, mas não diferiu devido ao tamanho da saída da extrusora $(p<0,1)$. Conclui-se que o processo de extrusão aplicado ao capim-maralfalfa neste experimento melhora a digestibilidade da matéria seca e da fibra em detergente neutro.

Palavras chave: Bagaço; Biomassa; Deslignificação; Fibra; In vitro. 


\section{Introducción}

En los rumiantes, y en general en los herbívoros, la base estructural de la alimentación son las plantas forrajeras - principalmente los pastos -, las cuales, dependiendo del sistema y del nivel de producción, en ocasiones se deben acompañar de otros alimentos con los cuales se espera atender los requerimientos de nutrientes y energía. En los países tropicales generalmente los pastos se ofrecen frescos, ya sea cosechados por el animal en el pastoreo o recolectados por el hombre y entregados en los comederos. No obstante, que existen opciones de conservación de los pastos como el heno o el ensilaje, éstas son de uso limitado en función de la fase fisiológica del animal, su nivel de producción, época del año y las posibilidades de vinculación al sistema de producción. El pasto maralfalfa (Pennisetum sp) es una gramínea tropical C4 de alto potencial de producción de biomasa que es utilizado crudo en la alimentación de bovinos en condiciones tropicales. Este pasto puede superar las 150 Ton de materia seca/año al tratarse de un pasto de porte alto de crecimiento erecto que puede alcanzar más de $165 \mathrm{~cm}$ de altura y superar los $440 \mathrm{~cm} 2$ de área foliar/planta. Su origen es aún incierto así como la forma en que fue introducido en Colombia. Sin embargo, se presume que se trata de un Pennisetum hybridum conocido en Brasil como Elefante Paraíso Matsuda [1]. Dicha gramínea fue el producto de la hibridación del Pennisetum americanum (L.) Leeke que aportó su calidad nutricional con el P. purpureum Schum que aportó su alto rendimiento de materia seca [2].

Al tratarse de una planta $\mathrm{C} 4$, posee un alto contenido de paredes celulares que se incrementan con la edad de corte y que pueden representar más del $60 \%$ de la materia seca a los 63 días de edad, pero que afectan la digestibilidad de la materia seca [3]. Según Clavero y Razz [3], la digestibilidad in vitro de la materia seca de esta gramínea se reduce de $62,5 \%$ a los 21 día de rebrote, a $52,1 \%$ a los 63 días de edad. En los rumiantes esta característica limita la capacidad de consumo de la dieta, el aporte de energía metabolizable y, con ello, la producción de leche y carne [4]. Para las condiciones tropicales se han utilizado diferentes alternativas que buscan mejorar la DMS de la dieta y la producción animal, siendo la más utilizada la suplementación con alimentos con alto contenido en almidón o en proteína o en ambos [5], pero paradójicamente ésta puede reducir la DMS del forraje [6] y encarecer el sistema de producción [7]. La baja digestibilidad de las paredes celulares es un problema que también afecta el uso de biomasas vegetales para la obtención de alcohol carburante de segunda generación [8]. Para superar este problema, se han propuesto diversos tipos de tratamientos que han sido agrupados en químicos, microbiológicos y físicos [9]. Entre estos últimos, la extrusión ha surgido como un proceso innovador que, al emplear presión, temperatura y fuerza de cizalla sobre las biomasas vegetales, tiene la capacidad de separarla en una fracción líquida (jugo) y otra sólida (bagazo fibroso), en la que se modifica la estructura de las paredes celulares [10] y se afecta la hidrólisis de la materia seca y de las paredes celulares [11,12], efectos que están poco documentados en gramíneas como el pasto maralfalfa. En alimentos para aves de postura existe más documentación que indica que la extrusión es uno de los métodos utilizados para reducir la actividad de los factores antinutricionales, incorporar efectos positivos en la digestibilidad de las proteínas al inactivar los inhibidores de las enzimas y desnaturalizar la proteína que se puede exponer a los sitios de ataque enzimático, además puede ampliar tanto la digestibilidad in vivo como in vitro del almidón [13]. No obstante, aunque existe evidencia de la aplicación de la extrusión sobre el mejoramiento del valor nutritivo de los alimentos, es limitada la información de la aplicación de este tipo de procesos en gramíneas como la maralfalfa y sus efectos. En virtud de lo anterior, se diseñó este experimento con el objetivo de evaluar la aplicación del proceso de extrusión en el pasto maralfalfa fresco sobre la composición de las paredes celulares evaluada por la metodología de Van Soest y sobre la digestibilidad in vitro de la materia seca (DIVMS) y de las paredes celulares (fibra en detergente neutro) (DIVFDN) con pepsinacelulasa, bajo la premisa que si dicho proceso ha modificado la estructura de dichas paredes [10] y afectado la hidrólisis de la materia seca y de las paredes celulares [11,12] en otras gramíneas, debería presentarse un comportamiento semejante en el pasto Pennisetum sp.

\section{Metodología}

En un predio del municipio de Támesis (Antioquia) localizado a $2200 \mathrm{msnm}$, se recolectaron siete muestras $(10,0 \mathrm{~kg} /$ muestra) de pasto maralfalfa 
de un solo lote con 51 días de rebrote que fue manejado homogéneamente durante la etapa de rebrote previa; las muestras frescas fueron picadas a $2 \mathrm{~cm}$ en una pica-pasto Yunke \#5. De cada muestra se tomó una submuestra de $200 \mathrm{~g}$ con la cual se conformó una muestra compuesta de pasto crudo: tres muestras frescas seleccionadas al azar fueron procesadas en un extrusor de tornillo cónico (prototipo diseñado por uno del coautores y fabricado localmente) con salida de $3 \mathrm{~mm}$ que giraba a $1050 \mathrm{rpm}$, mientras que las otras cuatro fueron sometidas al mismo proceso de extrusión, pero con salida de $1 \mathrm{~mm}$. Estos dos tamaños de salida fueron seleccionados con base en ensayos previos de calibración del equipo al tratarse de un prototipo fabricado localmente. En la Figura 1 se registra una fotografía del extrusor utilizado, con la información de sus condiciones de operación. Tanto en la muestra del pasto crudo seco como en los bagazos obtenidos luego de la extrusión, se evaluó el tamaño de la partícula utilizando tamices \# 4, 10, 14 y 20 (Pinzuar Ltda, Colombia), siguiendo el procedimiento manual descrito por Tayyab et al. [14]. Este consistió en armar el juego de tamices en orden de mayor a menor tamaño de orificio y colocar las muestras completas (previamente pesadas) en el tamiz superior (con mayor tamaño de orificio \#4). A continuación, el juego de tamices se sacudió cinco veces de izquierda a derecha sobre la superficie plana de una mesa de laboratorio y luego cinco veces de adelante hacia atrás. Después, se retiraron los tamices y se pesó el material retenido en cada tamiz con lo que se calculó el porcentaje másico de retención de partículas en cada tamiz.

Así mismo, tanto en la muestra compuesta del pasto sin procesar (crudo) como en los bagazos procedentes de la extrusión se determinó el contenido de fibra en detergente neutro (FDN) con base en el método oficial AOAC [15], fibra en detergente ácido (FDA) de acuerdo con el método oficial AOAC [16] y lignina en detergente ácido (LDA) implementado como método interno con base en Van Soest [17] y Van Soest y Wine [18]. Finalmente se determinó la digestibilidad in vitro (hidrólisis con pepsina-celulasa) de la materia seca (DIVMS) siguiendo la metodología descrita por López y Roldán [19] y de la FDN (DIVFDN). Todas las determinaciones se realizaron de acuerdo con los procedimientos del Laboratorio de Análisis Químico y Bromatológico de la Universidad Nacional de Colombia Sede Medellín.

El análisis estadístico de los resultados se realizó con la prueba T de Student de dos colas. El primer grupo de análisis confrontó los dos tratamientos con extrusión (salida de 1 y $3 \mathrm{~mm}$ ); en el segundo grupo la comparación fue entre el pasto crudo tomado como referencia - y los bagazos extruidos, en virtud de lo cual se consideró como un análisis de calidad de proceso $[20,21]$.

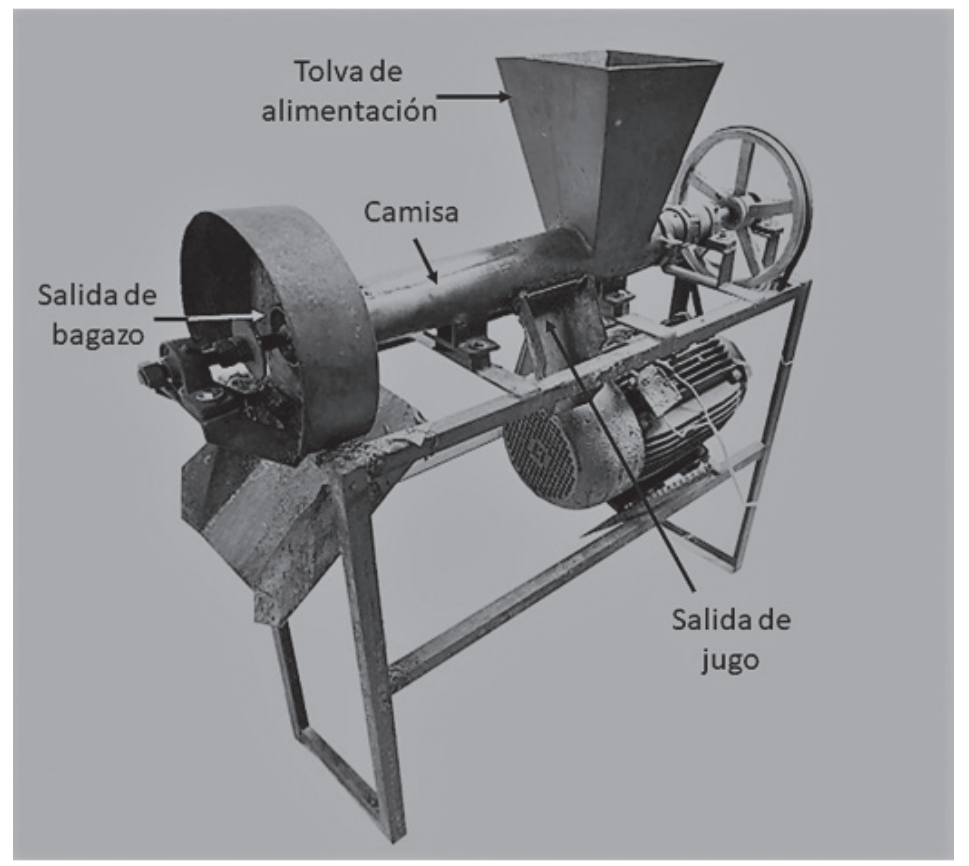

Figura 1. Fotografía del extrusor utilizado para el procesamiento de las muestras del pasto Pennisetum sp. Capacidad de procesamiento de $80 \mathrm{~kg}$ de MV/h [motor de $220 \mathrm{~V} ; 7.5 \mathrm{HP} ; 1050 \mathrm{rpm}$ ] (fotografía tomada por los autores). 


\section{Resultados y discusión}

En la Figura 2 se presenta el aspecto de las muestras de pasto crudo y de los bagazos luego del proceso de extrusión en donde se puede observar las diferencias en cuanto al tamaño de partículas y de textura. a

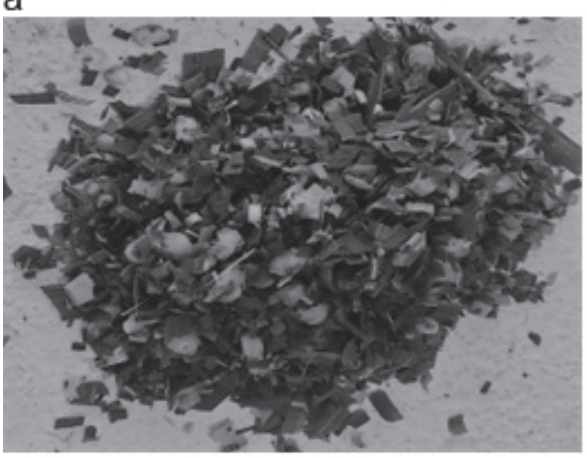

b

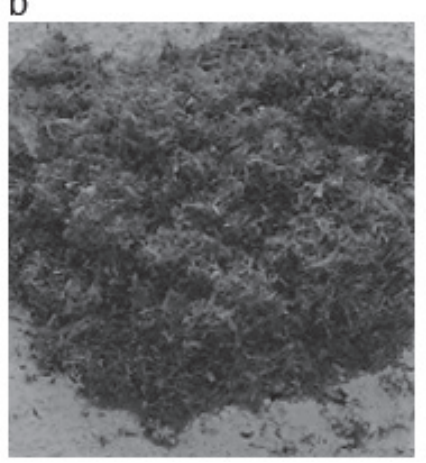

C

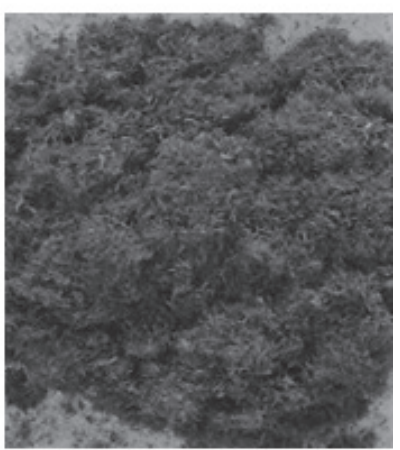

Figura 2. Foto del aspecto del pasto maralfalfa crudo picado (a), del bagazo generado por la extrusión con salida de 3 (b) y $1 \mathrm{~mm}$ (c).

En la Tabla 1 se presentan los resultados de la distribución del tamaño de la partícula en la masa de las muestras del pasto crudo y de los bagazos obtenidos por la aplicación de la extrusión con los dos tamaños de salida. El método para determinar la distribución del tamaño de la partícula en forrajes propuesto por Heinrichs y Kononoff [22] discrimina las partículas por su diámetro y longitud, toda vez que permite su paso cuando el tamaño es inferior al del poro de una determinada criba, lo cual puede suceder con partículas cuya longitud supera el diámetro del poro, pero su diámetro es inferior al del poro.

Tabla 1. Distribución del porcentaje másico del tamaño de la partícula retenida del pasto maralfalfa crudo picado y de los bagazos de la extrusión con dos tamaños de salida.

\begin{tabular}{|c|c|c|c|c|c|c|c|}
\hline \multirow{2}{*}{$\begin{array}{c}\text { Número de criba y tamaño } \\
\text { de la partícula } \\
3 \mathrm{~mm}\end{array}$} & \multirow{2}{*}{$\begin{array}{l}\text { Pasto } \\
\text { crudo }\end{array}$} & \multicolumn{2}{|c|}{$\begin{array}{c}\text { Bagazo del pasto } \\
\text { extruido }\end{array}$} & \multirow[t]{2}{*}{ Promedio } & \multirow[t]{2}{*}{ EEM $^{1}$} & \multirow{2}{*}{$p_{1}$} & \multirow{2}{*}{$p_{2}$} \\
\hline & & $3 \mathrm{~mm}$ & $1 \mathrm{~mm}$ & & & & \\
\hline $4(4,75 \mathrm{~mm})$ & 52,7 & 25,3 & 19,9 & 22,2 & 5,32 & 0,027 & 8,73E-08 \\
\hline $10(2,0 \mathrm{~mm})$ & 41,7 & 58,6 & 62,4 & 60,8 & 5,49 & 0,082 & $6,04 \mathrm{E}-07$ \\
\hline $14(1,4 \mathrm{~mm})$ & 2,48 & 4,17 & 4,75 & 4,50 & 0,225 & 0,175 & 0,01 \\
\hline $20(850 \mathrm{~mm})$ & 2,05 & 6,54 & 7,03 & 6,81 & 0,249 & 0,255 & 5,52E-08 \\
\hline Fondo & 1,13 & 5,42 & 5,90 & 5,69 & 0,427 & 0,374 & $3,29 \mathrm{E}-07$ \\
\hline
\end{tabular}

EEM $^{1}$ : Error estándar de la media; p1: probabilidad de establecer diferencia entre $3 \mathrm{~mm}$ y $1 \mathrm{~mm}$; p2: probabilidad de identificar diferencia entre el pasto crudo y los bagazos a $3 \mathrm{~mm}$ y $1 \mathrm{~mm}$.

En el presente estudio fue diferente la distribución de los tamaños de partícula en el pasto crudo y en los bagazos generados por la extrusión, así como entre los dos tamaños de salida del extrusor. En el primer caso, los resultados indican que en la criba \#4 se presentó una mayor retención de las partículas del pasto crudo que de los bagazos, pero en la criba \#10 se retuvo más del $58 \%$ de las partículas superiores a $2 \mathrm{~mm}$ de los bagazos extruidos. Williams et al [23] reportaron, igualmente, un incremento en la proporción de partículas de menor tamaño en el bagazo de paja de trigo y ensilaje de maíz obtenidos luego de ser sometidos a un proceso de extrusión. Esto pudo ser debido a que la fuerza de cizallamiento que ejerce el extrusor, genera el rompimiento de las partículas de alimento reduciendo su tamaño. Dicha fuerza de cizallamiento podría haberse 
incrementado con la reducción de la salida del extrusor como se pudo observar al comparar la distribución másica del tamaño de partículas entre los dos tamaños de salidas del extrusor. Mientras que con la salida de $3 \mathrm{~mm}$ se retuvo el $58,6 \%$ de las partículas, con la salida de $1 \mathrm{~mm}$ este valor aumento hasta $62,4 \%$.

La determinación de la distribución del tamaño de partícula en alimentos fibrosos para rumiantes constituye una información crítica en su manejo alimenticio debido a su relación con la incidencia de acidosis ruminal y laminitis, así como con la reducción en la concentración de grasa láctea, el consumo de materia seca y de la digestibilidad en la fibra [24]. Mertens [25] estableció que el tamaño de partícula crítico para vacas lactantes de alta producción es de $1,18 \mathrm{~mm}$, valor que utilizó en el método de laboratorio que propuso para estimar la FDN físicamente efectiva (peNDF, por sus siglas en inglés). De acuerdo con este autor, la peNDF se relaciona con las características de la fibra, principalmente con el tamaño de la partícula, la cual influye sobre la masticación y la naturaleza en dos fases de los contenidos ruminales (la flotación de la capa de grandes partículas en un conglomerado de líquido y partículas pequeñas). El método de laboratorio propuesto para estimar la peNDF se basó en tres supuestos: 1) que la FDN se distribuye de manera uniforme entre todas las partículas, 2) que la actividad de la masticación es igual en todas las partículas retenidas en la malla de $1,18 \mathrm{~mm}$ y 3 ) que no hay diferencia entre las fuentes de la fragilidad o la reducción en el tamaño de la partícula. Poppi et al [26], concluyeron que aquellos tamaños de partícula retenidos en las mallas a $1,18 \mathrm{~mm}$ tienen elevada resistencia a pasar por el rumen de bovinos y ovinos. En el presente trabajo se pudo establecer que más del $80 \%$ de las partículas del bagazo del pasto maralfalfa extruido a 1 ó $3 \mathrm{~mm}$, presentaron tamaños superiores a $1,18 \mathrm{~mm}$ (suma de los porcentajes de cribas \#4 y \#10), lo que sugeriría que el consumo de este tipo de bagazo no se asociaría con los problemas surgidos por el consumo de alimentos cuyos tamaños de partículas sean muy bajos, como los mencionados por el NRC [24]. Siguiendo a Poppi et al [26], es posible que las partículas de los bagazos generados en el proceso de extrusión en este estudio, permanezcan más tiempo en el rumen, característica que estaría relacionada con su degradabilidad en asociación con el nivel de FDN y de su composición en el alimento.
La Tabla 2 presenta los resultados de la composición química del pasto maralfalfa crudo y de los bagazos obtenidos con el proceso de extrusión donde se observa que el contenido de MS en estos últimos corresponde a 2.3-2.4 veces el del pasto crudo, indicando una alta capacidad de extracción de jugo por parte del equipo utilizado. Así mismo, se aprecia que al realizar el proceso de extrusión con una salida de $1 \mathrm{~mm}$, generó un aumento de solo $4,4 \%$ frente al proceso con la salida de $3 \mathrm{~mm}$ $(p<0.07)$ sugiriendo que no hay ventaja con la salida de $1 \mathrm{~mm}$. Por otro lado, se puede observar que el pasto crudo presentó alto contenido de FND, FDA y LDA con valores que superan a los reportados en trabajos anteriores realizados por Correa et al. [1] y por Correa [27] en los que se identificaron cifras inferiores al $60 \%$ de la MS para la FDN. Es probable que estas diferencias se deban a que el pasto utilizado en este ensayo fue obtenido de un predio cuyo manejo agronómico no implicaba el uso de fertilizantes químicos y ha sido demostrado que el contenido de FDN en pastos tropicales se incrementa significativamente en la medida en que se reduce la fertilización nitrogenada $[28,29]$.

En la misma tabla se identifica que no hubo diferencia en el contenido de FDN, FDA y LDA en los bagazos obtenidos con las diferentes salidas de la extrusión ( $p>0,1)$; sin embargo, hubo diferencia entre el pasto crudo y los bagazos generados por la extrusión. Mientras en los bagazos fue superior el contenido de FDN, el de FDA y LDA fue menor, lo cual sugeriría que hubo algún grado de modificación en la composición química de la pared celular debido a los efectos físicos generados por la extrusión como fue reportado por Zhan et al. [30].

En la Tabla 3 se presentan los resultados de los análisis de la DIVMS y la DIVFDN. Como se puede observar, la extrusión húmeda del pasto evaluado incrementó de manera significativa estos dos parámetros $(p<0,001)$ en $8,74 \pm 1,67 \%$ y $20,8 \pm$ $6,48 \%$, respectivamente, en comparación con el pasto crudo. Este efecto puede ser consecuencia de los cambios físicos y químicos que sufren los materiales lignocelulósicos que son sometidos al proceso de extrusión [30]. Es así como Heredia et al. [31] reportaron una reducción en la cristalinidad de la celulosa en el bagazo de sorgo dulce (Sorghum bicolor) debido a la extrusión, con lo que se mejoró la pososidad y susceptibilidad de las paredes celulares a la acción de las enzimas fibrolíticas. Barakat et al. [32] señalan, así mismo, 
que este tipo de procesos mecánicos no solo reducen el tamaño de partícula y la cristalinidad de la celulosa si no que, además, contribuye a la disociación de los tejidos vegetales y a la ruptura de la pared celular que, en conjunto, mejoran la digestibilidad de la biomasa vegetal. Por otro lado,
Duque et al [33] han indicado que la extrusión afecta los enlaces entre lignina y hemicelulosa con la celulosa con lo que se mejora el acceso de las enzimas celulolíticas $\mathrm{y}$, con ello, la digestibilidad de la celulosa.

Tabla 2. Porcentaje de materia seca, fibra en detergente neutro (FDN), fibra en detergente ácido (FDA) y lignina en detergente ácido (LDA) del pasto maralfalfa crudo y de los bagazos obtenidos a partir de la extrusión con dos tamaños de salida (Valores expresados en $\mathrm{g} / 100 \mathrm{~g}$ de materia seca).

\begin{tabular}{cccccccc}
\hline \multirow{2}{*}{$\begin{array}{c}\text { Fracción } \\
\text { química }\end{array}$} & $\begin{array}{c}\text { Pasto } \\
\text { crudo }\end{array}$ & $\begin{array}{c}\text { Bagazo del pasto } \\
\text { extruido }\end{array}$ & Promedio & EEM ${ }^{1}$ & $\mathbf{p}_{1}$ & $\mathbf{p}_{\mathbf{2}}$ \\
\cline { 3 - 4 } & & $\mathbf{3} \mathbf{~ m m}$ & $\mathbf{1} \mathbf{~ m m}$ & & & & \\
\hline MS & 23,0 & 52,7 & 55,0 & 54,0 & 1,733 & 0,07 & $2 \mathrm{E}-09$ \\
FDN & 73,4 & 79,3 & 80,1 & 79,8 & 0,307 & 0,140 & $2 \mathrm{E}-07$ \\
FDA & 47,7 & 46,9 & 46,2 & 46,5 & 0,475 & 0,246 & $4 \mathrm{E}-03$ \\
LDA & 5,70 & 5,23 & 4,85 & 5,01 & 0,103 & 0,179 & $2 \mathrm{E}-03$ \\
\hline
\end{tabular}

${ }^{1}$ EEM: Error estándar de la media; $p_{1}$ : probabilidad de la diferencia entre $3 \mathrm{~mm}$ y $1 \mathrm{~mm} ; \mathrm{p}_{2}$ : probabilidad de la diferencia entre el pasto crudo y los bagazos a $3 \mathrm{~mm}$ y $1 \mathrm{~mm}$

Existen pocos registros en la literatura en los que se haya evaluado el efecto de la extrusión sobre la digestibilidad de materiales lignocelulósicos en rumiantes. Sanders [34] reportó aumento en la DIVMS de la semilla de algodón con linter procesada en un extrusor de un solo tornillo, pero no la afectó en la semilla de algodón sin linter. En un trabajo más reciente, Elgemark [11] estableció aumento en la DIVMS y la DIVFDN del ensilaje del pasto Pheleum pratense $\mathrm{L}$ y de Trifolium pratense $\mathrm{L}$ sometidos a extrusión en estado semi-seco indicando que dicho proceso afectó la estructura física y química de estos materiales.

Tabla 3. Digestibilidad in vitro de la materia seca (DIVMS) y de la fibra en detergente neutro del pasto (DIVFDN) maralfalfa crudo y del bagazo producido por la extrusión con dos tamaños de salida (Valores expresados en g/100 g).

\begin{tabular}{cccccccc}
\hline \multirow{2}{*}{ Digestibilidad } & $\begin{array}{c}\text { Pasto } \\
\text { crudo }\end{array}$ & \multicolumn{2}{c}{ Bagazo del pasto extruido } & Promedio & EEM $^{1}$ & $\boldsymbol{p}_{1}$ & $\boldsymbol{p}_{2}$ \\
\cline { 3 - 4 } & $\mathbf{3} \mathbf{~ m m}$ & $\mathbf{1} \mathbf{~ m m}$ & 48,9 & 48,5 & 0,47 & 0,174 & $9 \mathrm{E}-06$ \\
DIVMS & 44,6 & 48,1 & $46,2^{\mathrm{a}}$ & 44,8 & 3,16 & 0,058 & 0,001 \\
\hline DIVFDN & 37,1 & $42,9^{\mathrm{b}}$ & &
\end{tabular}

${ }^{1}$ EEM: Error estándar de la media; $p_{1}$ : probabilidad de la diferencia entre $3 \mathrm{~mm}$ y $1 \mathrm{~mm}$; $\mathrm{p}_{2}$ : probabilidad de la diferencia entre el pasto crudo y los bagazos a $3 \mathrm{~mm}$ y $1 \mathrm{~mm}$

a,bValores con letras diferentes en la misma línea son diferentes

Kononoff et al. [35], por su parte, establecieron que la reducción del tamaño de la partícula en el ensilaje de maíz aumentó la fermentabilidad de la materia seca en el rumen, pero no afectó el pH ruminal, la producción y el contenido de grasa en la leche. En el presente trabajo se pudo establecer una correlación negativa y significativa entre la proporción de partículas retenidas en la criba \#4 - la que retendría partículas de mayor tamaño con la DIVMS $(r=-0,389 ; p<0,01)$ y la DIVFDN $(=$ $-0,736 ; p<0,001)$. En la criba \#10 fue diferente el resultado: la correlación fue positiva y significativa entre la proporción de las partículas retenidas con la DIVMS $(r=0,373 ; p<0,01)$ y la DIVFDN $(=0,712$; $p<0,001)$. Estos resultados indican que la reducción en el tamaño de las partículas - que se consigue con la criba \#10 aumentaría la digestibilidad de la MS y de la FDN. Este tipo de respuestas deberá evaluarse en condiciones in vivo antes de llegar a conclusiones concretas al respecto.

En el procesamiento de biomasas vegetales para la obtención de alcohol carburante de segunda generación, el tamaño de partícula es igualmente importante puesto que se ha demostrado que existe una relación inversa entre el tamaño de partícula y la eficiencia en el proceso de hidrólisis enzimática 
[36] como fue hallada en este trabajo, tal y como se esperaría que suceda en el rumen [37].

Finalmente, en este estudio se pudo establecer una correlación alta y positiva entre la DIVMS y la DIVFDN $(r=0,859 ; p<0,01)$ indicando que la técnica in vitro basada en el uso de la pepsina-celulasa permite evaluar apropiadamente la digestibilidad de las paredes celulares de materiales lignocelulósicos. Esta correlación es de esperarse si se tiene en cuenta que en gramíneas, como la utilizada en este estudio, la mayor proporción de la materia seca está constituida por paredes celulares, que en el estudio estuvieron representadas por la FDN. En este panorama se podría esperar que la DIVMS y la DIVFDN varíen de manera paralela y por ello, que los valores de digestibilidad se encuentren alta y significativamente asociadas. Este tipo de correlaciones las han reportado otros autores $[37,38]$ haciendo de la técnica in vitro enzimática una alternativa metodológica confiable, poco dispendiosa y de bajo costo.

\section{Conclusiones}

Este experimento se realizó con el objetivo de evaluar la aplicación del proceso de extrusión en el pasto maralfalfa fresco sobre la composición de las paredes celulares evaluada por la metodología de Van Soest y sobre la digestibilidad in vitro de la materia seca (DIVMS). Los resultados del estudio mostraron que la extrusión del pasto húmedo efectivamente modificó la composición química de las paredes celulares en el bagazo e incrementó la DIVMS y la DIVFDN.

En el mismo estudio se estableció que la reducción en el tamaño de la salida del extrusor no afectó la digestibilidad del pasto maralfalfa, pero modificó la distribución de las partículas en las cribas de 4,75 y de $2,0 \mathrm{~mm}$. Se estableció que más del $80 \%$ de las partículas de los bagazos fue mayor a 1,18 $\mathrm{mm}$, tamaño que es crítico en la alimentación de rumiantes. Finalmente, se estableció una correlación negativa entre el tamaño de la partícula y la DIVMS y la DIVFDN lo cual favorece la utilización de este material en la alimentación de rumiantes.

\section{Referencias bibliográficas}

[1] Correa HJ, Ceron JM, Arroyave H, Henao Y, López A. Pasto Maralfalfa: mitos y realidades. En: Cooperativa Colanta, IV seminario internacional Competitividad en carne y leche,
2004 nov 10-11; Medellín, Colombia. 2004. p. 231-274.

[2] Hanna WW, Gaines TP, Gonzales B, Monson WG. Effects of ploid on yield and quality of pearl millet $x$ napiergrass hybrids. Agron J. 1984;76:669-971.

[3] Clavero T, Razz R. Valor nutritivo del pasto maralfalfa (Pennisetum purpureum $x$ Pennisetum glaucum) en condiciones de defoliación. Revista Facultad de Agronomía (online). 2009;26(1):78-87.

[4] Kolver ES. Nutritional limitations to increased production on pasture based systems. Proceedings of the Nutrition Society, 2003;62:291-300.

[5] Natsir A. Fibre utilization by ruminants. Masagena Press, Makassar. 2012. 243 p.

[6] Ferraretto LF, Shaver RD. Making sense of starch by NDF interactions. In: Penn State Dairy Nutrition Workshop. Proceedings of the Penn State Dairy Nutrition Workshop. Grantville, PA, United States of America. 2016. p. 45-50.

[7] CONPES (Consejo Nacional de Política Económica y Social). Política Nacional Para Mejorar La Competitividad Del Sector Lácteo Colombiano. República de Colombia, Departamento Nacional de Planeación, 2010.

[8] dos Santos AC, Ximenes E, Kim Y, Ladisch M. Lignin-Enzyme Interactions in the Hydrolysis of Lignocellulosic Biomass. Trends in Biotechnology. 2019;37(5):518-531.

[9] Agbor VB, Cicek N, Sparling R, Berlin A, Levin DB. Biomass pretreatment: Fundamentals toward application. Biotechnology Advances. 2011;29:675-685.

[10]Lin Z, Liu L, Li R, Shi J. Screw Extrusion Pretreatments to Enhance the Hydrolysis of Lignocellulosic Biomass. J Micr Bioch Tech. 2012;5(S12):5.

[11] Elgemark E. Intensively processed silage using Bio-extruder. Uppsala, Sweden: Swedish University of Agricultural Sciences, Department of Animal Nutrition and Management; 2019.

[12] Sanders KJ. The effects of extrusion on ruminal digestion and performance of ruminants. Lubbock, TX, United States of America: Texas Tech University; 1998.

[13] Lichovnikovaa M, Zemanb L, Kracmarb S, Kleckera $D$. The effect of the extrusion process on the digestibility of feed given to laying hens. Anim Feed Sci and Tech. 2004;116:313-318.

[14] Tayyab U, Wilkinson RG, Reynolds CK, 
Sinclair LA. Particle size distribution of forages and mixed rations, and their relationship with ration variability and performance of UK dairy herds. Liv Sci. 2018;217:108-115.

[15]AOAC. Official method 2002.04. Amylasetreated neutral detergent fiber in feeds using refluxng in beakers or crucibles. First action 2002. Final action 2005.

[16]AOAC. official method 973.18. Fiber (acid detergent) and lignin $\mathrm{H}_{2} \mathrm{SO}_{4}$ in animal feed. First action 1977. Final action 1977.

[17] Van Soest PJ. Use of detergent in analysis of fibrous feeds. III. Study of effects of heating and drying on yield of fiber and Lignin Forage. J AOAC. 1965;48(4):787-790.

[18] Van Soest PJ; Wine, RH. Determination of lignin and cellulose in acid detergent fiber with permanganate. J AOAC. 1968;51(4):780785.

[19]López HA, Roldan M. Estandarización del método de la celulasa para la determinación de la digestibilidad in vitro (trabajo de grado). Medellín, Colombia: Universidad Nacional de Colombia, Facultad de Ciencias Agropecuarias; 1991.

[20]Ross A, Willson VL. One-Sample T-Test. In: Basic and Advanced Statistical Tests. Rotterdam: Sense Publishers; 2017. p. 9-12.

[21]Feng $Y$, Huang $Y, M a ~ X$. The application of Student'st-test in internal quality control of clinical laboratory. Front Lab Med. 2017;1:125128.

[22] Heinrichs J, Kononoff P. Evaluating Particle Size of Forages and TMRs using the New Penn State Forage Particle Separator. DAS 02- 42. Pennsylvania State University, University Park, PA, United States of America; 2002.

[23] Williams A, van der Poel A, Boer $\mathrm{H}$, Tamminga $S$. The Effect of Extrusion Conditions on the Fermentability of Wheat Straw and Corn Silage. J Sci Food Agr. Vol. 1999;74:117-124.

[24] National Research Council (NRC) 2001 Nutrient Requirements of Dairy Cattle. Seventh Revised Edition. National Academy Press, Washington, D C. 405 p.

[25] Mertens DR. Creating a system for meeting the fiber requirements of dairy cows. Journal of Dairy Science. 1997;80:1463-1481.

[26] Poppi DP, Hendricksen RE, Minson DJ. The relative resistance to escape of leaf and stem particles from the rumen of cattle and sheep. J.
Agric. Sci. 1985;105:9-14.

[27] Correa HJ. Calidad nutricional del pasto maralfalfa (Pennisetum sp) cosechado a dos edades de rebrote. Livestock Research for Rural Development. 2006;18(6):326-335.

[28] Delevatti LM, Cardoso AS, Barbero RP, Leite LG, Romanzini EP, Ruggieri A, Reis RA. Effect of nitrogen application rate on yield, forage quality, and animal performance in a tropical pasture. Sci Rep. 2019;9(1):7596.

[29] Hassan A, Zewdu T, Urge M, Fikru S. Effect of Nitrogen Fertilizer Application on Nutritive Value of Cenchrus ciliaris and Panicum Maximum Grown under Irrigation at Gode, Somali Region. J Nutr Food Sci. 2015;S11:S11005.

[30]Zhan X, Wang D, Bean SR, Mo X, Sun XS, Boyle D. Ethanol production from supercriticalfluid-extrusion cooked sorghum. Industrial Crops and Products. 2006;23(3):304-310.

[31] Heredia-Olea E, Pérez-Carrillo E, MontoyaChiw M, Serna-Saldívar SO. Effects of extrusion pretreatment parameters on sweet sorghum bagasse enzymatic hydrolysis and its subsequent conversion into bioethanol. Biomed Res Int. 2015;2015:325905.

[32] Barakat A, Mayer C, Solhy A, Arancon RAD, De Vries $\mathrm{H}$, Luque R. Mechanical pretreatments of lignocellulosic biomass: towards facile and environmentally sound technologies for biofuels production. RSC Adv. 2014;4:4810948127.

[33]Duque A, Manzanares $P$, Ballesteros $M$. Extrusion as a pretreatment for lignocellulosic biomass: Fundamentals and applications. Renew Energy. 2017;114:1427-1441.

[34] Sanders KJ. The effects of extrusion on ruminal digestion and performance of ruminants. Lubbock, Texas, United States of America: Texas Tech University; 1998.

[35] Kononoff PJ, Heinrichs AJ, Lehman HA. The Effect of Corn Silage Particle Size on Eating Behavior, Chewing Activities, and Rumen Fermentation in Lactating Dairy Cows. Journal of Dairy Science. 2003;86(10):3343-3353.

[36] Khullar E, Dien BS, Rausch KD, Tumbleson ME, Singh V. Effect of particle size on enzymatic hydrolysis of pretreated Miscanthus. Industrial Crops and Products. 2013;44,11-17.

[37] Bellet N, Besle J and Demarquilly C 1998 Ammonia treatment of lucerne and cocksfoot harvested at two growth stages: Effect 
on cell wall composition and digestibility, Champanelle, Francia. $19 \mathrm{p}$.

[38] Gosselink JMJ, Dulphy JP, Poncet C, Jailler M, Tamminga S, Cone JW. Prediction of forage digestibility in ruminants using in situ and in vitro techniques. Animal Feed Science and Technology. 2004;115(3-4):227-246. 